\title{
On the distribution of Campsicnemus armatus (Zetterstedt, 1849) species group (Diptera: Dolichopodidae)
}

\author{
I.Ya. Grichanov \\ All-Russian Institute of Plant Protection \\ Podbelskogo 3, St. Petersburg-Pushkin, 196608. Russia. \\ E-mail: grichanov@mail.ru
}

The Campsicnemus armatus species group differs from the other groups in simple male legs, but with the mid tibia bearing a comb-like posteroventral row of blunt-ended bristles. It includes the Palaearctic Campsicnemus armatus (Zetterstedt, 1849), C. pumilio (Zetterstedt, 1843), C. vtorovi Negrobov et Zlobin, 1978, and C. caffer Curran, 1926, known from northern and southern Africa. C. armatus var. deserti Vaillant, 1953 (unavailable name) from Algeria is associated with $C$. caffer, which is now spread in the two zoogeographical Regions. New records are given for C. armatus, C. vtorovi and C. caffer. Modified couplets in a key to Palearctic species of Campsicnemus and a new key to Afrotropical species of the genus are provided. Photographs of male antenna and mid tibia of species of the Campsicnemus armatus group are published for the first time.

Key words: Diptera, Dolichopodidae, Palaearctic Region, Afrotropical Region, Campsicnemus armatus species group, new records, key.

\section{Introduction}

The genus Campsicnemus Haliday in Walker et al., 1851, has an extremely high diversity of endemic species in the Hawaiian Islands and French Polynesia (Evenhuis, 2009; 2015), with more than 310 species worldwide (Grichanov, 2017a). Grichanov (1998; 2009) composed the last identification keys to the Afrotropical and Palaearctic species of Campsicnemus. C. armatus (Zetterstedt, 1849) was included into the both keys, as well as into the key to Campsicnemus males from Atlantic Ocean islands (Grichanov, 2012a). The last paper has proposed to consider the southern African C. caffer Curran, 1926 as a subspecies of the trans-Palaearctic $C$. armatus, which has also two very close Palaearctic species, i.e. the trans-Palaearctic C. pumilio (Zetterstedt, 1843) and C. vtorovi Negrobov et Zlobin, 1978 known only from its type locality in Kyrgyzstan.

Grichanov (2012a) has described three new Afrotropical species of the genus and excluded $C$. a. armatus and $C$. atlanticus Dyte, 1980 from the Region. For this study, I have re-examined the recently published material on $C$. armatus, $C$. caffer, C. pumilio and C. vtorovi(Grichanov, 2012b, 2017b; Grichanov et al., 2017; Grichanov, Bagachanova, 2018; Grichanov, Ovsyannikova, 2018) and provide new records. Modified couplets in a key to Palearctic species of Campsicnemus and a new key to Afrotropical species of the genus are also presented, and characters of the $C$. armatusgroup of species are discussed and illustrated.

\section{Material and methods}

The material examined is housed at the National Museum, Bloemfontein, South Africa (BMSA), the Natal Museum, Pietermaritzburg, South Africa (NMSA), the Museum of Zoology, University of Lund, Sweden (MZLU), the Zoological Institute of the Russian Academy of Sciences, St. Petersburg, Russia (ZIN) and the Zoological Museum of Moscow State University, Moscow, Russia (ZMUM). The material published previously and collected from Iran, Russia (Arkhangelsk, Murmansk, Voronezh, Ulyanovsk Regions, Altai Territory, Yakutia), Sweden, South Africa has been re-examined (but not listed here).

Specimens were studied and photographed with a ZEISS Discovery V-12 stereo microscope and an AxioCam MRc5 camera. Morphological terminology and abbreviations follow Grichanov, Brooks (2017). 


\section{Results}

\section{Campsicnemus armatus species group}

DIAGNOSIS. Male legs simple, but the mid tibia bearing a comb-like posteroventral row of blunt-ended bristles.

Campsicnemus armatus (Zetterstedt, 1849)

(Figs 1, 4, 9, 10)
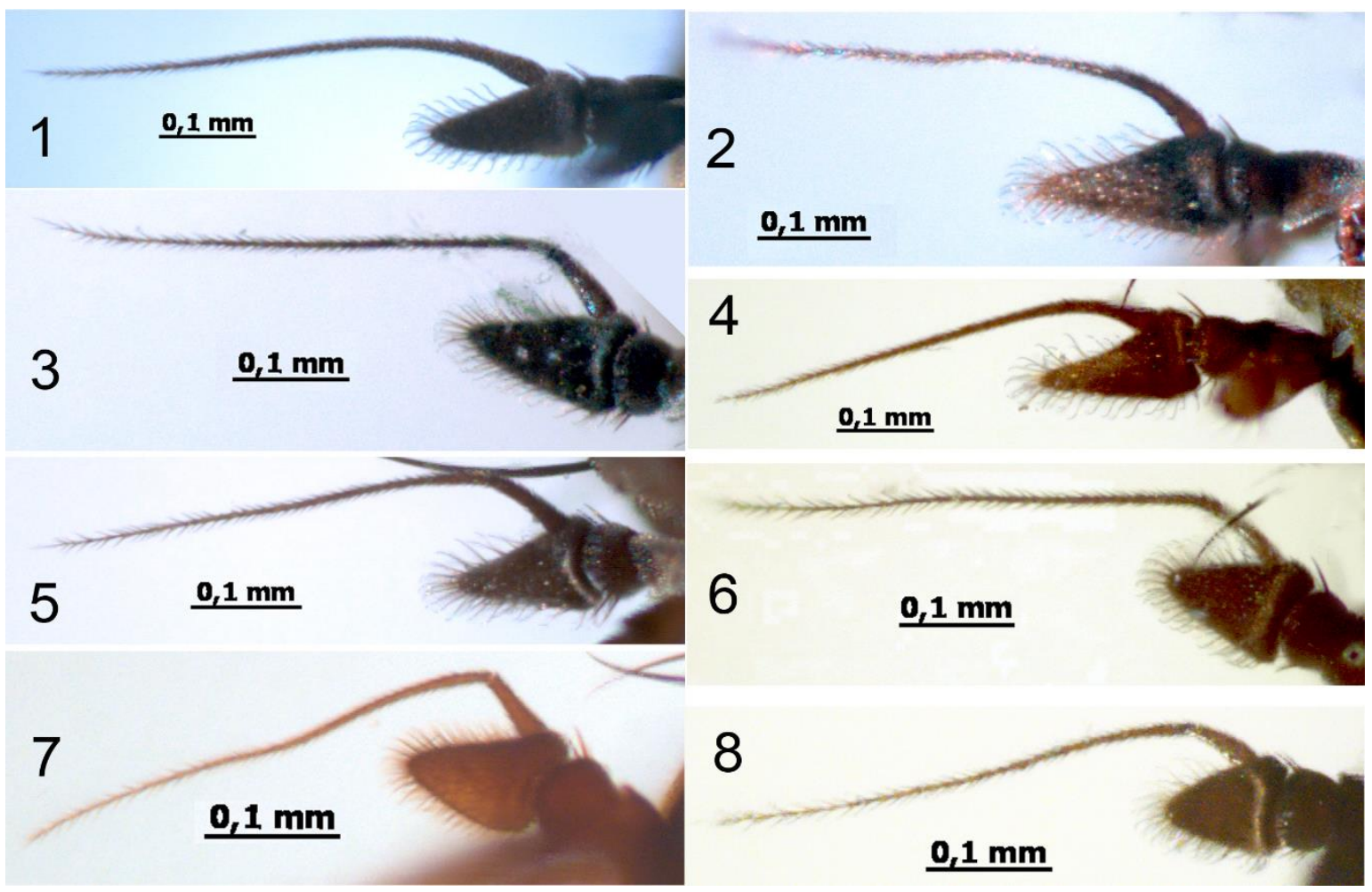

Figs 1-8. Campsicnemus sp., male antenna. Figs 1, 4. C. armatus (Zetterstedt): 1 - Russia: Solovetskie Islands; 4 - Russia: NE Yakutia. Figs 2, 3, 5. C. vtorovi Negrobov et Zlobin: 2 - Russia: Voronezh Region; 3 - Russia: Ulyanovsk Region; 5 Iran: Markazi Prov. Figs 6-8. C. cafferCurran: 6 - South Africa: Western Cape; 7 - South Africa: KwaZulu-Natal (in ethanol); 8 - Ethiopia: Oromia.

Dolichopus armatus Zetterstedt, 1849: 3093. Type locality: Denmark: Rosenthal, Gryphium (male lectotype and 5 paralectotypes examined in MZLU)).

Campsicnemus prodromus (Haliday, 1832) (nec Meigen, 1824).

Camptosceles prodromus Haliday, 1832: 358. Type locality: Ireland: Holywood.

Campsicnemus pectinifer De Meijere, 1907: 178. Type locality: Netherlands: Diemen, Naarden.

MATERIAL. 10", [Russia: Nenetsia:] 60 km N Narjan-Mar, Pechora River, 10.VII.2008, N.Vikhrev (ZMUM).

DISTRIBUTION. Palaearctic: Austria, Belgium, Czech Republic, Denmark, Finland, France, Germany, Iceland, Ireland, Italy, Mongolia, Netherlands, Norway, Poland, Russia (Arkhangelsk, Kamchatka, Karelia, Krasnoyarsk, Murmansk, Nenetsia, Tatarstan, Yakutia, Yamal, Yekaterinburg), Slovakia, Sweden, Turkey (Kars), UK. Here excluded from Iran and North Africa.

Campsicnemus pumilio (Zetterstedt, 1843)

Dolichopus pumilio Zetterstedt, 1843: 606. Type locality: Sweden: "Sueciam in Scania ad Scandhammar et Silfakra" (male lectotype and 4 paralectotypes examined in MZLU, 2 more possible paralectotypes in Wallengren collection, MZLU).

Campsicnemus pectinulatus Loew, 1864: 390; Lundbeck, 1912: 368; Parent, 1938: 620, fig. 848. Type locality: Germany: "Ziegelwiese bei Halle"

MATERIAL. 10", Russia: Novgorod env., 4.07.2012, I. Grichanov (ZIN).

DISTRIBUTION. Palaearctic: Austria, Belgium, Czech, Denmark, Estonia, Finland, France, Germany, N Kazakhstan, Kyrgyzstan, Netherlands, Poland, Romania, Russia (Karelia, Krasnodar, Krasnoyarsk, Leningrad, Moscow, Novgorod, Ryazan, Yakutia, Kamchatka), Sweden, UK. Here excluded from Ulyanovsk Region.

\section{Campsicnemus vtorovi Negrobov et Zlobin, 1978}

(Figs 2, 3, 5, 11-14)

Campsicnemus vtorovi Negrobov et Zlobin, 1978: 54. Type locality: Kyrgyzstan: Naryn river valley, Karakolka Mt.

Campsicnemus pumilio Grichanov, 2012b: 251 (nec Zetterstedt, 1843; misid.).

Campsicnemus armatus Ahmadi et al., 2017: 68; Grichanov, 2017b: 36; Grichanov et al., 2017: 100 (nec Zetterstedt, 1849; misid.). 
MATERIAL. Iran: 20", Markazi prov.: Arak env., 26 km W, Baneh village, 3405' N, 49²4' E, 2117 m a.s.l., 19.V.2017; $10^{\circ}$, Shazand env., 7 km W, Astaneh Town env., Seidan chashmah, 3355' N, 49²0' E, 2289 m a.s.l., 20.V.2017; Russia: 10', Ulyanovsk Region, Sengilei Distr., Shilovka env., 1-3.IX.2010, K. Tomkovich (ZIN); 80̊, Voronezh Region, Liski Distr., Divnogor'e, 8, 9.VII.1994, Zlobin (ZIN).

DISTRIBUTION. Palaearctic: Iran (Lorestan, Markazi), Kyrgyzstan, Russia (Altai Territory; Ulyanovsk, Voronezh). New for Iran and Altai Territory and Ulyanovsk Region of Russia.

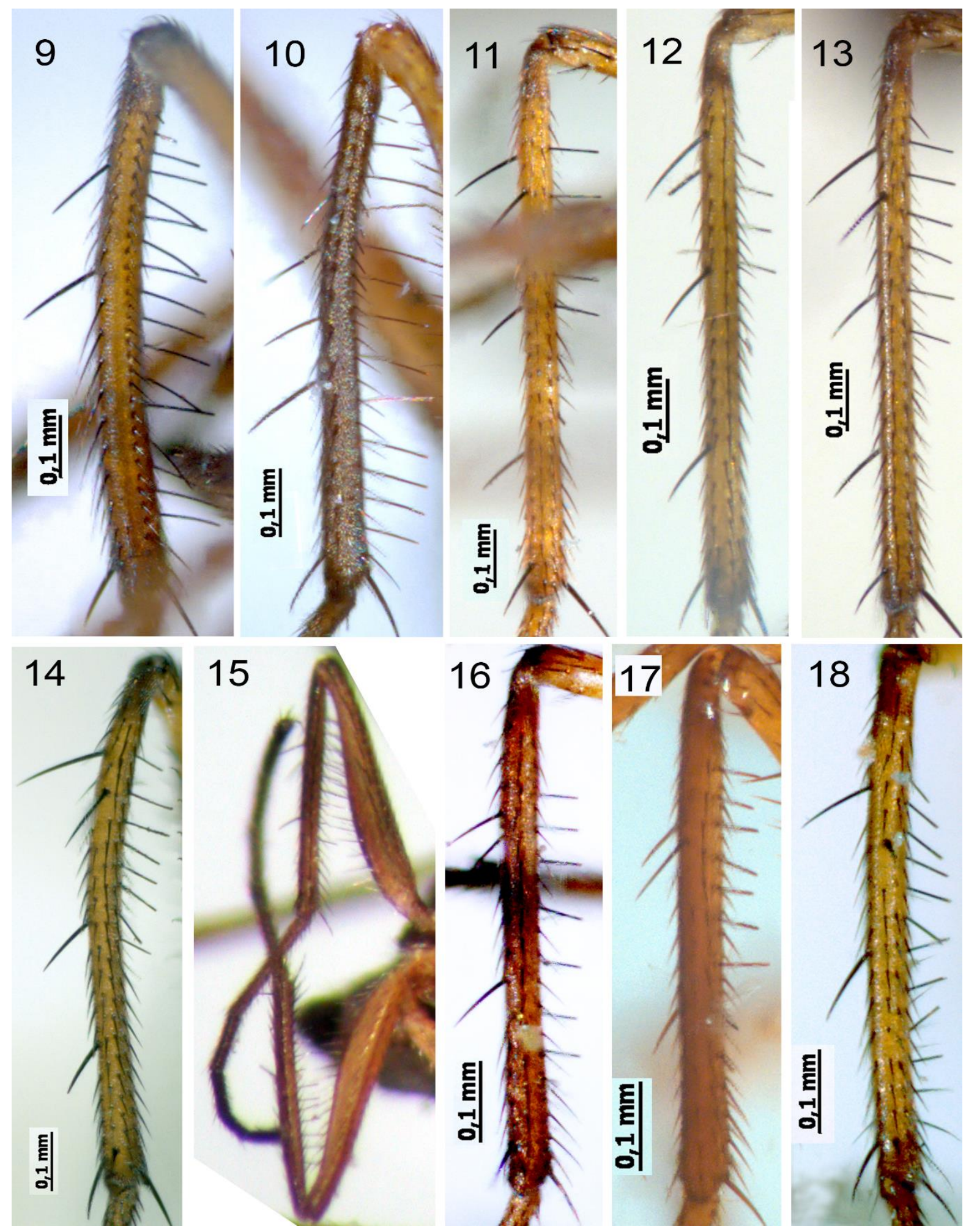

Figs 9-18. Campsicnemus sp., mid tibia. Figs 9, 10. C. armatus (Zetterstedt): 9 - Russia: Solovetskie Islands; 10 - Russia: NE Yakutia; Figs 11-14. C. vtorovi Negrobov et Zlobin: 11 - Russia: Voronezh Region; 12 - Russia: Ulyanovsk Region; 13 Russia: Altai Territory; 14 - Iran: Markazi Prov. Figs 15-18. C. caffer Curran: 15 - South Africa: Mpumalanga (holotype); 16 - South Africa: Western Cape; 17 - South Africa: KwaZulu-Natal (in ethanol); 18 - Ethiopia: Oromia. 


\section{Campsicnemus caffer Curran, 1926}

(Figs 6-8, 15-18)

Campsicnemus caffer Curran, 1926: 15; Grichanov \& Mostovski, 2009a: 42. Type locality: South Africa: Mpumalanga: Barberton (male lectotype and 1 paralectotype examined in NMSA).

Campsicnemus armatus caffer Curran, 1926; Grichanov, 2012a: 8.

Campsicnemus armatus deserti Vaillant, 1953: 11 (as var. of C. armatus); Negrobov, 1991: 59 (as a subsp. of C. armatus, unavailable name according to ICZN, 45.6.4.1, as published after 1980). Type locality: not given [SE Algeria: Tassili n'Ajjer].

MATERIAL. Ethiopia: 20", Oromia, Ziwai L., $7.91^{\circ} \mathrm{N}, 38.73^{\circ} \mathrm{E}, 1640 \mathrm{~m}, 11-13.11 .2012, \mathrm{~N}$. Vikhrev (ZMUM); Namibia: 29, Katima Mulilo Distr., Salambala Forest at: 17050.066'S / 24³6.225'E, 926 m, Malaise trap, 18-20.XI.2012, 926 m, A.H. KirkSpriggs / Miombo and Moppane woodlands (BMSA); South Africa: 10', Free State, Brandfort, Florisbad Res. Stat., 2846.039'S, 2604.234'E, 25-29.X.2010, A.H. Kirk-Spriggs / Malaise traps, Acacia savanna (BMSA); 19, Free State,Harrismith Scotland Farm, $27^{\circ} 28.595^{\prime}$ S, 29³7.098'E, 10-12.XI.2009, A.H. Kirk-Spriggs / Malaise traps, dense Leucosedea dominated scrub (BMSA); 20', 19, Western Cape, Gamkaskloof (Die Hel), 33²1.808'S, 2137.650'E, 16-18.X.2012, 336 m, A.H. Kirk-Spriggs / Malaise traps, Karoo and valley, Acacia woodland (BMSA); 19, same label, but 12-13.IX.2013; $10^{\text {' }}$ (in ethanol), KZN, Royal Natal Nature Reserve, Gudu Forest, 2840.90'S, 2855.78'E, 1680-1730m, 22.9-17.XI.2006, Mostovski M.B., MT (NMSA).

DISTRIBUTION. Afrotropical: Ethiopia, Namibia, South Africa; Palaearctic: Algeria. First record from Ethiopia.

Modified couplets in the key to Palearctic species of Campsicnemus (males) published by Grichanov (2009)

33. Mid tibia without a comb-like ventral row of blunt-ended bristles, with rows of simple hairs and setae; mid basitarsus

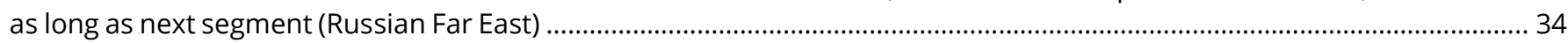
- Mid tibia with a comb-like posteroventral row of blunt-ended bristles; mid basitarsus distinctly longer than next segment

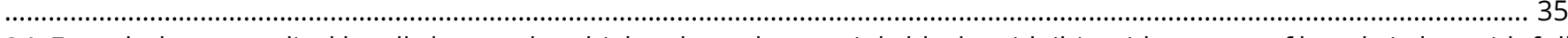

34. Face dark; postpedicel hardly longer than high at base; legs mainly black; mid tibia without row of long bristles, with full row of erect posteroventral setae, nearly as long as diameter of tibia; $2.3 \mathrm{~mm}$ C. zlobiniGrichanov, 2012 - Face silvery white; postpedicel 2 times longer than high at base; legs almost completely pale yellow; mid tibia with row of 7-8 bristles in distal 2/3, two times longer than diameter of tibia; $1.3-1.5 \mathrm{~mm}$....... C. versicolorus Negrobov et Zlobin, 1978 35. Fore coxa with only yellow hairs and bristles; mid tibia usually with 3 dorsal bristles; antennal postpedicel triangular, slightly longer than high at base; $1.5 \mathrm{~mm}$ (northern and southern Africa) caffer Curran, 1926

- Fore coxa with at least some apical bristles black; mid tibia usually with 4 dorsal bristles; antennal postpedicel triangular or elongate triangular.....

37. Antennal postpedicel triangular, slightly longer than high at base, with rounded apex; mid tibia with a comb-like row of blunt-ended bristles in basal half only; mid femur usually with 1 row of ventral setae; 1.5 (Trans-Palaearctic except subarctic) pumilio (Zetterstedt, 1843)

- Antennal postpedicel elongate triangular, nearly twice longer than high at base, with acute apex; other features various

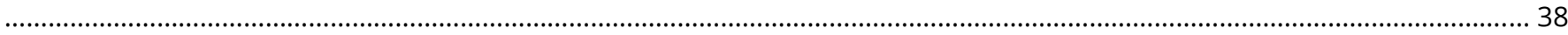

38. Mid tibia, posteroventrally along almost whole length, with a comb-like row of blunt-ended bristles; mid femur usually with 2 rows of ventral setae; 1.5-2.0 (Trans-Palaearctic except subtropics) ..................................... armatus (Zetterstedt, 1849) - Mid tibia with a comb-like row of blunt-ended bristles in basal half only; 1.7-2.4 mm (Iran, Kyrgyzstan, Russia) vtorovi Negrobov et Zlobin, 1978.

Key to Afrotropical species of Campsicnemus (males)*

*Three species and subspecies ( $C$. a. armatus, $C$. a. deserti and $C$. atlanticus) are excluded from the last key (Grichanov, 1998), and three species described from the St. Helena Island (Grichanov, 2012a) are added.

1. Antenna mostly brownish yellow; fore leg simple; mid femur simple or modified; mid tibia more or less strongly thickened,

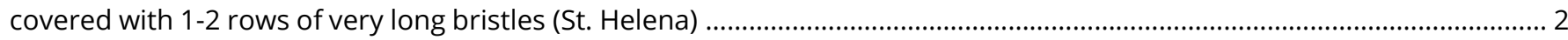

- Antenna black; fore leg simple or modified; mid femur simple; mid tibia simple, without very long bristles ....................... 6

3. Mid tibia considerably dilated along entire length and curved; mid basitarsus with thick apical tooth; $1.5 \mathrm{~mm}$ sanctaehelenae Grichanov, 2012

- Mid tibia not dilated or slightly thickened; mid basitarsus with at most thin apical spine

4. Mid femur without ventral subapical excavation; mid tibia with erect ventral spinules and cilia along entire length; mid basitarsus 1.5 times longer than next segment; $3.7 \mathrm{~mm}$.... flavissimus Grichanov, 2012

- Mid femur with deep ventral subapical excavation; mid tibia without erect ventral spinules and cilia; mid basitarsus no longer than next segment

5. Mid tibia and basitarsus densely covered with long setae along entire length; antennal postpedicel 3 times longer than high at base, with drawn-out apex; $1.85 \mathrm{~mm}$ meridionalis Grichanov, 2012 6. Fore tibia strongly dilated; tarsal segments 1, 2 and 4 shortened, and 1st-3rd segments of fore tarsus bearing very long processes covered with long hairs; $3.0 \mathrm{~mm}$ (St. Helena; Palaearctic) magius (Loew, 1845)

- Fore tibia simple or slightly dilated; tarsal segments simple or variously ornamented .... 7

7. Fore tibia swollen, with dorsal row of 4 long setae on basal half; fore basitarsus with long appendages; other tarsomeres with long setae; about $2 \mathrm{~mm}$ (DR Congo) yangiGrichanov, 1998

- Fore tibia simple; fore basitarsus without appendages 8 
8. Fore coxa with black hairs and bristles; mid tibia with row of simple ventral hairs; fore tarsomeres 1-3 with 2 rows of long, 3-4 times as long as diameter of segments; fore basitarsus with one long bristle at base reaching tip of $2^{\text {nd }}$ segment; 2.5 $\mathrm{mm}$ (Cameroon) lantsoviGrichanov, 1998

- Fore coxa with yellow hairs and bristles; mid tibia with posteroventral row of blunt black setae; fore tarsus without long setae; $1.5 \mathrm{~mm}$ (northern and southern Africa)..... cafferCurran, 1926

\section{Discussion}

The European authors (Parent, 1938; Negrobov, Stackelberg, 1969; d'Assis Fonseca, 1978; etc.) distinguished Campsicnemus armatus and $C$. pumilio ( $=$ C. pectinulatus) by the relative length of antennal postpedicel and the number of blunt bristles on mid tibia mainly. Negrobov \& Zlobin (1978) described a new species $C$. vtorovi from mountains of Kyrgyzstan, comparing it with $C$. pectinulatus. C. vtorovishares features of both $C$. armatus and $C$. pumilio, having elongate triangular postpedicel and comb-like row of blunt-ended bristles in basal half of mid tibia. This morphospecies was most probably overlooked in Europe until recently. All other characters used in those species descriptions and in keys seem to be variable, including colour of the legs (mostly yellow or mostly blackish femora and tibiae). Nevertheless, the number and length of posteroventral blunt-ended bristles are also variable in all the three species (see figs 9-18 and references below).

The most common species of the group, $C$. armatus and $C$. pumilio, have been often reported from the same countries (Austria, Belgium, Czech Republic, Denmark, Finland, France, Germany, Netherlands, Poland, Sweden, UK) and territories of Russia (Kamchatka, Karelia, Krasnoyarsk, Yakutia). This distributional pattern along with the variability of mid leg setation suggests that the two forms are both of one species. C. pumilio is also characterized by somewhat shorter antennal postpedicel, similar to that in $C$. caffer (see below), but this is also a variable character (figs 1-8). Taking into the consideration the new records of the intermediate $C$. vtorovi in this paper, it cannot be regarded as a subspecies of either C. armatus or C. pumilio.

Curran (1926) distinguished his new species from $C$. armatus by the presence of only three dorsal bristles on mid tibia (four bristles are usually present in the other species of the group). However, the number, position, and length of these bristles seem to be variable. Most C. armatus specimens examined have mid tibia with four bristles (a pair of postero/anterodorsals on basal third and a pair of dorsals below). Nevertheless, at least one male and one female of the latter species from the Russian North (Nenetsia and Solovetskie Islands) have mid tibia with only three bristles (a pair of postero/anterodorsals on basal third and one dorsal below). Vaillant (1953) distinguished his central Saharan variety from the European specimens of $C$. armatus by entirely yellow bristles on fore coxa, and this character seems to be present in all Afrotropical specimens examined. The discovery of $C$. caffer in mountains of Ethiopia has allowed me to associate the desertivariety sensu Vaillant with this South-African species, rather than with $C$. armatus. The characters briefly described by Vaillant (1953) for the desertivariety are identical with those in $C$. caffer. The Palaearctic specimens of $C$. armatus, $C$. pumilio and $C$. vtorovi bear 2 to 6 black bristles on fore coxa, sometimes with addition of a few yellow or golden bristles. The number and length of ventral bristles on mid femur and tibia are variable in $C$. caffer as well. Generally, these bristles are somewhat weaker in $C$. caffer (as in C. pumilio and C. vtorovi), than in C. armatus. The antennal postpedicel of $C$. caffer males is shorter than that in males of $C$. armatus and $C$. vtorovi. In addition, $C$. armatus variety with dark or blackish femora and tibiae is not uncommon (see Parent, 1938), while the similar variety is yet unknown in $C$. caffer.

Species of the Campsicnemus armatus group seem to be rather variable in its wide area, being a multiregional polyzonal species or a complex of cryptic species distinguished poorly without molecular analysis. C. caffer has a peculiar disjunctive area, occurring in southern (SAR and Namibia) and northern Africa (SE Algeria and Ethiopia). It is now spread formally in two zoogeographical Regions, though the species was reported in mountains of Algeria not far from the border with the Republic of the Niger. The nominotypical species C. armatus is distributed almost all over the boreal band of Palaearctic Region (except subtropics), inhabiting the banks of various water sources.

Campsicnemus as a whole is poorly represented in the Afrotropics, with three species on the continent and four species on the St. Helena, which were probably introduced to the Island by human. This is true for at least $C$. magius, mainly Mediterranean species. The continental African species of the genus have close relations with some groups of much richer Palaearctic fauna (with about 40 species).

\section{Acknowledgments}

I am greatly indebted to Drs. Ashley H. Kirk-Spriggs (BMSA), Mike Mostovski (NMSA), Roy Danielsson (MZLU), N.E. Vikhrev and A.L. Ozerov (ZMUM) for their kindness in furnishing an opportunity to study the collections of their Museums. The work is performed within the Program for Basic Scientific Researches in the Russian Federation for the Long-Term Period (2013-2020) approved by Order No. 2538-r of the Government of the Russian Federation dated 27 December 2012 , and supported by the All-Russian Institute of Plant Protection project N 0665-2019-0014. 


\section{References}

Ahmadi, A., Gheibi, M., Ostovan, H., Hesami, S., \& Grichanov, I.Ya. (2017). New records of long-legged flies (Diptera, Dolichopodidae) of Iran. Russian Entomological Journal, 26(1), 65-70. Available http://kmkjournals.com/journals/REJ/REJ_Index_Volumes/REJ26/REJ_26_1065 070 Ahmadi et al (accessed 15 May 2019 ).

d'Assis Fonseca, E.C.M. (1978). Handbooks for the identification of British insects, Volume 9, part 5: Diptera Orthorrhapha Brachycera, Dolichopodidae. Royal Entomological Society of London, 1-90.

Curran, C.H. (1926). Records of African Dolichopodidae with descriptions of new species. Revue zoologique africaine, 14(1), 1-39.

De Meijere, J.C.H. (1907). Eerste Supplement op de nieuvve. Naam lijst van Nederlandsche Diptera. Tijdschrift voor Entomologie, 50(4), 151195. Available from: https://www.biodiversitylibrary.org/bibliography/10088\#/summary (accessed 15 May 2019).

Evenhuis, N.L. (2009). Review of Campsicnemus (Diptera: Dolichopodidae) of the Marquesas, French Polynesia, with description of four new species groups. Zootaxa, 2004, 25-48.

Evenhuis, N.L. (2015). New species of Campsicnemus Haliday (Diptera: Dolichopodidae) from Moloka'i, Hawaiian Islands. In: N.L. Evenhuis and, S.E. Miller (eds.), Records of the Hawaii Biological Survey for 2014. Part I: Articles. Bishop Museum Occasional Papers, $116,3-17$. Available from: http://hbs.bishopmuseum.org/pubs-online/pdf/op116p3-17.pdf (accessed 15 May 2019).

Grichanov, I.Ya. (1998). Two new species of Campsicnemus Haliday (Diptera: Dolichopodidae) from tropical Africa. International Journal of Dipterological Research, 9, 109-113.

Grichanov, I.Ya. (2009). A new species of Campsicnemus Haliday from Azerbaijan with a key to the Palearctic species of the genus (Diptera: Dolichopodidae). Far Eastern Entomologist, 198, 1-16. Available from: http://www.biosoil.ru/FEE/Publication/282 (accessed 15 May 2019 ). Grichanov, I.Ya. (2012a). Review of Campsicnemus species from the Atlantic Ocean islands (Diptera: Dolichopodidae). European Journal of Taxonomy, 11, 1-12. DOI: https://doi.org/10.5852/ejt.2012.11 (accessed 15 May 2019).

Grichanov, I.Ya. (2012b). A new species of Campsicnemus from the Far East of Russia with some new records (Dolichopodidae, Diptera). Amurian zoological journal, 4(3), 250-252.

Grichanov, I.Ya. (2017a). Alphabetic list of generic and specific names of predatory flies of the epifamily Dolichopodoidae (Diptera). 2nd Edition. St.Petersburg: VIZR, 1-563. (Plant Protection News Supplements, N23). Available from: https://archive.org/download/Grichanov2017DolibankSec/Grichanov\%202017\%20Dolibank-sec.pdf (accessed 15 May 2019).

Grichanov, I.Ya. (2017b). First data on Dolichopodidae (Diptera) of the Tigirekskii Nature Reserve (Altai Territory, Russia). Acta Biologica Sibirica, 3 (1), 34-39. http://dx.doi.org/10.14258/abs.v3i1.2181.

Grichanov, I.Ya., Ahmadi, A., \& Kosterin, O.E. (2017). New records of long-legged flies (Diptera, Dolichopodidae) from Central and NorthEastern Iran. Acta Biologica Sibirica, 3(4), 99-112. DOI: https://doi.org/10.14258/abs.v3i4.3636 (accessed 15 May 2019).

Grichanov, I.Ya., Bagachanova, A.K. (2018). An annotated checklist of Dolichopodidae (Diptera) of Yakutia (Siberia) with some new records. Russian Entomological Journal, 27(1), 73-92. Available http://kmkjournals.com/journals/REJ/REJ Index Volumes/REJ 27/REJ 271073092 Grichanov Bagachanova (accessed 15 May 2019 ).

Grichanov, I.Ya., Brooks, S.E. (2017). 56. Dolichopodidae (long-legged dance flies). In: Kirk-Spriggs, A.H., Sinclair, B.J. (Eds), Manual of Afrotropical Diptera. Volume 2. Nematocerous Diptera and lower Brachycera. Suricata 5. Pretoria: SANBI Graphics \& Editing, $1265-1320$. Available from: http://afrotropicalmanual.org (accessed 15 May 2019).

Grichanov, I.Ya., Mostovski, M.B. (2009). Long-legged flies (Diptera: Dolichopodidae) in the collection of the Natal Museum: a review of C. H. Curran's types, new synonyms, and new combinations. Zootaxa, 2194, 37-53.

Grichanov, I.Ya., Ovsyannikova, E.I. (2018). Notes on Dolichopodidae (Diptera) from the White Sea coast and islands (Russian North). Acta Biologica Sibirica, 4 (4), 19-24. http://dx.doi.org/10.14258/abs.

Haliday, A.H. (1832). The characters of two new dipterous genera, with indications of some generic subdivisions and several undescribed species of Dolichopodidae. The Zoological Journal London, 5 [1830-1831], 350-367.

Loew, H. (1864). Ueber die in der zweiten Hälfte des Juli 1864 auf der Ziegelwiese bei Halle beobachteten Dipteren. Zeitschrift für die Gesammten Naturwissenschaften, 24, 377-396.

Lundbeck, W. (1912). Diptera Danica. Genera and species of flies hitherto found in Denmark. Part 4, Dolichopodidae. Gad, Copenhagen, 1414. Available from: https://www.biodiversitylibrary.org/item/35313\#page/9/mode/1up (accessed 15 May 2019).

Negrobov, O.P. (1991). Dolichopodidae. In: Soos, A., Papp, L., Oosterbroeck, P. (Eds.). Catalogue of Palaearctic Diptera 7: DolichopodidaePlatypezidae. Budapest: Akadémiai Kiadó, 1-291. DOI:10.1016/B978-0-444-98731-0.50008-9.

Negrobov, O.P., Stackelberg, A.A. (1969). Family Dolichopodidae. In: Bei-Bienko, G.Ya. (Ed.). Keys to the Fauna of Euriopean Parts of USSR, 5(1). Leningrad: Nauka: 670-751 (in Russian).

Negrobov, O.P., Zlobin, V.V. (1978). A review of species of the genus Campsicnemus Walker (Diptera, Dolichopodidae) in the USSR fauna. Nauchnye doklady vysshei shkoly. Biologicheskie nauki, 1, 51-59 (in Russian).

Parent, O. (1938). Diptères dolichopodides. Faune de France, 35, 1-720. Available from: http://faunedefrance.org/bibliotheque/docs/O.PARENT(FdeFr35)Dipt.Dolochopodidae.pdf (accessed 15 May 2019).

Vaillant, F. (1953). Sur quelques Dolichopodidae du Tassili n'Ajjer. Mission scientifique en Tassili des Ajjer (1949): 1. Recherches zoologiques et medicales. Les fourmis du Tassili des Ajjer (Sahara central). Institut de Recherches Sahariennes de I'Université d'Alger, Algeria, 3-18.

Zetterstedt, J.W. (1843). Diptera Scandinaviae disposita et descripta. Officina Lundbergiana, Lundae [= Lund], 2, 441-894.

Zetterstedt, J.W. (1849). Diptera Scandinaviae disposita et descripta. Officina Lundbergiana, Lundae [= Lund], 8, 2935-3366.

\section{Citation:}

Grichanov I.Ya. (2019). On the distribution of Campsicnemus armatus (Zetterstedt, 1849) species group (Diptera: Dolichopodidae). Acta Biologica Sibirica, 5 (2), 145-150.

Submitted: 14.04.2019. Accepted: 15.06.2019

cross ref http://dx.doi.org/ 10.14258/abs.v5.i2.6210

(C) 2019 by the authors. Submitted for possible open access publication under the terms and conditions of the

Creative Commons Attribution (CC BY) license (http://creativecommons.org/licenses/by/4.0/). 